\title{
The hazards of honey: infantile botulism
}

\author{
Jennifer K Smith, ${ }^{1}$ Sarah Burns, ${ }^{2}$ Steve Cunningham, ${ }^{3}$ Julie Freeman, ${ }^{2}$ Ailsa McLellan, ${ }^{4}$ \\ Kenneth McWilliam
}

${ }^{1}$ Department of Paediatrics, RHSC Edinburgh, Edinburgh, UK

${ }^{2}$ PICU, RHSC Edinburgh, Edinburgh, UK

${ }^{3}$ Department of Respiratory and Sleep Medicine, RHSC Edinburgh, Edinburgh, UK

${ }^{4}$ Department of Neurology, RHSC Edinburgh, Edinburgh, UK

Correspondence to Jennifer K Smith, jennifer.k.smith@luht.scot.nhs.uk

\section{Summary}

Infantile botulism is a rare cause of neuromuscular weakness resulting from ingestion of Clostridium botulinum—an anaerobic Gram-positive bacillus found universally in soil. The only definite food source known to cause infantile botulism is honey; previously, links to formula milk have been postulated but not definitely sourced. We present an interesting case report of a 2-month-old infant with this rare condition, including the diagnostic difficulties that ensued. A brief overview of the condition follows. This is the first case in the UK in which $C$ botulinum was successfully isolated from both the patient and the suspected source-a jar of honey. The importance of food labelling as a public health message is highlighted.

\section{BACKGROUND}

Infantile botulism is rare in the UK. Only 10 cases have been reported between 1976 and 2009; all of which have required admission to hospital. Approximately $90 \%$ of cases worldwide occur in those younger than 6 months old.

This case highlights the diagnostic challenge of infantile botulism. In the absence of classical electromyographic findings, a diagnosis of botulism was not considered likely and the infant was thought to have neurodegenerative process that would quickly lead to death. Only when the electromyogram (EMG) was repeated showing a different pattern was a stool sample sent and withdrawal of care measures postponed. The therapeutic window for treatment had long passed by the time a diagnosis was reached.

Clinicians must consider infantile botulism in the differential diagnosis of weakness in infancy and send an appropriate stool sample for PCR early in the clinical course. Diagnosis may be very difficult, particularly if EMG studies are not classical, and treatment should be started as early as possible if botulism is clinically suspected.

Labelling of honey jars was first implemented in 1996; however, there continues to be a need for greater public awareness of the harmful effects of honey to infants less than 1 year.

\section{CASE PRESENTATION}

A term, previously well, male infant presented to his hospital aged 2 months with a 6-day history of poor feeding, constipation and irritability. He was less interactive with poor fixing and following. He was dehydrated on examination and had fluid resuscitation and antibiotics for presumed sepsis.

He was a thriving, breastfed infant with a regular bowel pattern. Of note, there was a background social history of maternal heroin use in the first trimester and ongoing methadone dependency.
He was transferred to the regional tertiary neurology centre for investigation of generalised weakness. He was found to be globally hypotonic with poor head control and spontaneous but weak movement of all four limbs. There were upper but no lower limb reflexes. He had a poor suck, no rooting reflex and reduced gag reflex. He had no cry though vocalised weakly. Funduscopy revealed no papilloedema or retinal haemorrhages. Pupils were equal and reactive but he was unable to fix or follow.

He was started on gentamicin, cefotaxime, aciclovir and dexamethasone for a presumed diagnosis of encephalopathy. These were discontinued following negative cerebrospinal fluid and blood cultures. Nasopharyngeal secretions were positive for rhinovirus and a chest $\mathrm{x}$-ray (CXR) showed mild right-middle lobe opacification. Investigations for his neurological pathology are detailed in table 1 . He became pale and hypotonic a few days later necessitating admission to the high dependency unit. His CXR revealed ongoing right-middle zone changes and he was started on co-amoxiclav and continuous positive airway pressure (CPAP). He continued intermittently on CPAP for a number of days and appeared to show signs of improvement.

On day 16 of admission he had abnormal movements in keeping with a generalised tonic-clonic seizure and was treated with midazolam and phenytoin. His electroencephalography was entirely normal. A tensilon test was performed, which was also normal, and an EMG suggested central rather than peripheral weakness.

He was admitted to the paediatric intensive care unit (PICU) on day 32 following a profound apnoea and desaturation requiring intubation and ventilation. Given the deteriorating nature of his condition and negative investigations at that time, he was thought to have a neurodegenerative condition with a poor prognosis. End-of-life care was discussed with parents and arrangements made for baptism. 
Table 1 Investigations

\begin{tabular}{|c|c|c|}
\hline Investigations & & Results \\
\hline Blood & $\begin{array}{l}\text { FBC, U+Es, LFTs, ammonia, lactate, metabolic screen, amino acids, TFTs, culture, PT, } \\
\text { APTT, vitamin } B_{12} \text {, folate, VLCFA, biotinidase, acylcarnitine, osmolality, lysosomal } \\
\text { enzymes, B-galactosidase, A-galactosidase, desialylated transferrin }\end{array}$ & All within normal limits \\
\hline Urine & Amino acids, organic acids, toxicology, creatinine, osmolality & Normal \\
\hline Cerebrospinal fluid & $\begin{array}{l}\text { Culture, virology, protein, glucose, lactate, cell count, oligoclonal bands, glycine, serine, } \\
\text { opening pressure }\end{array}$ & All negative \\
\hline Respiratory secretions & Virology, bacteriology, H1N1 & Rhinovirus Escherichia coli \\
\hline Imaging & $\begin{array}{l}\text { Chest x-ray } \\
\text { Skeletal survey, abdominal USS, cranial USS, CT brain, MRI brain }{ }^{2}\end{array}$ & $\begin{array}{l}\text { Right-middle lobe consolidation } \\
\text { Normal }\end{array}$ \\
\hline Stool & PCR and culture & C botulinum type $\mathrm{A}$ isolated \\
\hline Sleep study & & Central apnoeas \\
\hline EEG & & Normal \\
\hline Neurophysiology & $\begin{array}{l}\text { Nerve conduction: left ulnar and median normal deltoid, extensor digitorum longus, first } \\
\text { dorsal interossei = no decremental response, no increased insertional activity, no spon- } \\
\text { taneous activity }\end{array}$ & $\begin{array}{l}\text { Central rather than peripheral weakness } \\
\text { suggested on first record. Reversed pat- } \\
\text { tern on repeat attempt }\end{array}$ \\
\hline
\end{tabular}

APTT, activated partial thromboplastin time; EEG, electroencephalography; U+Es, Urea and electrolytes; FBC, full blood count; LFTs, liver function tests; PT, prothrombin time; TFTs, thyroid function tests; USS, ultrasound scanning; VLCFA, very long chain fatty acids.

In attempts to reach a definitive diagnosis, a MRI scan was repeated and was again normal. Neurophysiology was repeated and revealed a changing pattern in keeping with a peripheral myopathic process. Therefore infantile botulism could not be excluded and a stool sample was sent to the national reference laboratory. On day 41 of admission, Clostridium botulinum type A was isolated by PCR on his stool sample. The diagnosis of infantile botulism was made and public health authorities informed. History from both parents revealed the infant had been getting honey on his soother at home. A sample of the honey from the jar used subsequently tested positive for $C$ botulinum type A. Given the duration of symptoms prior to diagnosis, the decision was made not to administer immunoglobulin.

Seven weeks after initial presentation the infant failed a trial of extubation and showed no signs of sustained neurological improvement having initially recovered some power in his limbs. A tracheostomy was performed and he remained dependant on CPAP ventilation 3 months after presentation. He continued to secrete $C$ botulinum in stool samples tested weekly.

\section{DIFFERENTIAL DIAGNOSIS}

Neurodegenerative process of uncertain aetiology.

\section{TREATMENT}

Treatment with human-derived botulinum anti-toxin (Baby BIG-IV) has been available in the USA since 2003 and was used in similar cases in the UK in 2007 and 2009. It is associated with fewer side effects, such as serum sickness, than traditionally used ovine derived anti-toxin. Current recommendations suggest not treating if symptom onset is greater than 10 days before diagnosis, as in this case. Treatment should be started if botulism is suspected without waiting for laboratory confirmation.

\section{OUTCOME AND FOLLOW-UP}

At the time of writing, the infant had been discharged home after successfully weaning off all respiratory support. He was assumed to have made a full recovery but will be followed up in the out-patient clinic as a matter of routine.

\section{DISCUSSION}

Infantile botulism is rare in the UK. Only 10 cases have been reported between 1976 and 2009; all of which have required admission to hospital. Approximately $90 \%$ of cases worldwide occur in those younger than 6 months old. ${ }^{1}$

Neurotoxin-producing $C$ botulinum spores are ingested, germinate then colonise the large intestine. Toxin is absorbed and binds to peripheral presynaptic nerve terminals inhibiting release of acetylcholine. It affects ganglionic synapses, parasympathetic synapses and neuromuscular junctions, which explains the clinical picture of descending flaccid paralysis and anticholinergic symptoms. Young infants are particularly vulnerable due to the susceptibility of their gut to colonisation due to immature intestinal flora and lack of Clostridium-inhibiting bile acids. This is in contrast to botulism in adults, which follows the ingestion of pre-formed toxin.

Symptoms present insidiously and are classically described as constipation, cranial nerve palsies and progressive generalised weakness. Constipation secondary to a paralytic ileus can pre-date the onset of weakness by up to 14 days. Other reported symptoms include poor feeding, weak cry, lethargy, seizures, dry mouth and papillary dilatation. Muscle contractions that require frequent neuromuscular transmission - that is, sucking, swallowing and head control-are often affected early and can be the last symptoms to improve. ${ }^{2}$

Diagnosis is made by identifying $C$ botulinum in stools supported by typical EMG findings. Positive EMG will classically demonstrate a triad of (1) small evoked compound muscle action potentials (CMAP) to supermaximal stimulation, (2) tetanic and post-tetanic facilitation of CMAPs in response to $20-50 \mathrm{~Hz}$ stimulation and (3) absence of post-tetanic exhaustion of CMAPs. ${ }^{3}$ Interpretation can be particularly difficult in young infants, leading to false negative results, as seen in this case. Particular care must be taken to stimulate nerves for the recommended 10 s. Absence of post-tetanic exhaustion is particularly specific for infantile botulism and should be looked for.

Treatment with Baby BIG-IV has been available in the USA since 2003. Underwood et al ${ }^{4}$ suggested a median 
hospital stay of 35 days (PICU stay of 24 days) for untreated patients in contrast to the treated group where median hospital stay was 15 days (PICU stay 9 days). There was no significant reduction in requirement for intubation or duration of mechanical ventilation. In this review, spanning 30 years, only two patients required a tracheostomy. All patients survived and with no serious sequelae.

At the age of 6 months this infant continues to be noninvasive ventilation dependent-a relatively long stay when compared to other reports in the literature. There is some evidence aminoglycoside use potentiates the neurotoxic effects of botulinum ${ }^{5}$; this patient received gentamicin at first presentation, which may account for the longer duration of symptoms.

\section{REFERENCES}

1. Health Protection Report. Vol 3, No 46. 20 November 2009

2. Cox N, Hinkle R. Infant botulism. Am Fam Physician 2002;65:1388-92.

3. Gutierrez AR, Bodensteiner J, Gutmann L. Electrodiagnosis of infantile botulism. J Child Neurol 1994;9:362-5.

4. Underwood K, Rubin S, Deakers T, et al. Infant botulism: a 30-year experience spanning the introduction of botulism immune globulin intravenous in the intensive care unit at Childrens Hospital Los Angeles. Pediatrics 2007; 120:e1380-5.

5. Santos JI, Swensen P, Glasgow LA. Potentiation of Clostridium botulinum toxin by aminoglycoside antibiotics: clinical and laboratory observations. Pediatrics 1981;68:50-4.

\section{Learning points}

- Infantile botulism must always be considered early in the differential diagnosis of weakness in infancy even if non-classical or even opposing EMG findings (as in this case) are demonstrated.

- Stool samples for $C$ botulinum PCR confirm the diagnosis easily and should be sent early as a basic investigation.

- Food sourcing is possible and, in this case, eliciting the honey-soother history proved invaluable. Such questions ought to be part of routine questioning of carers in such cases.

- Labelling of honey has been a requirement since 1996 in the UK but clearly this is not always enough to prevent administration of honey to infants under the age of 1 year. Therefore, this message must be imparted opportunistically by those working in the community with infants and their carers-specifically community midwives and health visitors.

\section{Competing interests None.}

Patient consent Obtained.

This pdf has been created automatically from the final edited text and images.

Copyright 2010 BMJ Publishing Group. All rights reserved. For permission to reuse any of this content visit http://group.bmj.com/group/rights-licensing/permissions.

BMJ Case Report Fellows may re-use this article for personal use and teaching without any further permission.

Please cite this article as follows (you will need to access the article online to obtain the date of publication).

Smith JK, Burns S, Cunningham S, Freeman J, McLellan A, McWilliam K. The hazards of honey: infantile botulism. BMJ Case Reports 2010;

10.1136/bcr.05.2010.3038, date of publication

Become a Fellow of BMJ Case Reports today and you can:

- Submit as many cases as you like

- Enjoy fast sympathetic peer review and rapid publication of accepted articles

- Access all the published articles

Re-use any of the published material for personal use and teaching without further permission

For information on Institutional Fellowships contact consortiasales@bmjgroup.com

Visit casereports.bmj.com for more articles like this and to become a Fellow 